\title{
Correlação entre capacidade funcional e qualidade de vida em idosas hipertensas submetidas a treinamento resistido
}

\section{Correlation between functional fitness and quality of life in elderly hipertensive women undergoing resistance training}

\author{
Juliana Fernandes de Souzali; Ivan Daniel Bezerra Nogueira²; Murillo Jales Lins de Lira ${ }^{3}$; Eline da \\ Cunha Silva4; Gardênia Maria Holanda Ferreira5; Patrícia Angélica de Miranda Silva Nogueira ${ }^{5}$ \\ ${ }^{1}$ Graduanda e bolsista da PROPESQ - Universidade Federal do Rio Grande do Norte - UFRN, Natal, Rio Grande do Norte, Brasil. \\ ${ }^{2}$ Professor assistente do Departamento de Fisioterapia - Universidade Federal do Rio Grande do Norte - UFRN, Natal, Rio Grande do \\ Norte, Brasil. \\ ${ }^{3}$ Graduando e bolsista da PROPESQ - Universidade Federal do Rio Grande do Norte - UFRN, Natal, Rio Grande do Norte, Brasil. \\ ${ }^{4}$ Mestre pelo programa de pós-graduação do Departamento de Fisioterapia - Universidade Federal do Rio Grande do Norte - UFRN, \\ Natal, Rio Grande do Norte, Brasil. \\ ${ }^{5}$ Professora adjunta do departamento de fisioterapia - Universidade Federal do Rio Grande do Norte - UFRN, Natal, Rio Grande do \\ Norte, Brasil.

\section{Endereço para correspondência} \\ Patrícia Angélica de Miranda Silva Nogueira \\ Rua Ataulfo Alves, nº 1904, Apto.1101 - Candelária \\ - Natal - RN \\ CEP: 59064-570 \\ idpa02@ufrnet.br / idpa01@hotmail.com
}

\begin{abstract}
Resumo
Introdução: A hipertensão arterial sistêmica exerce influência na dependência funcional e qualidade de vida dos idosos, sendo a distância percorrida no teste da caminhada de 6 minutos preconizada como alternativa para avaliação da capacidade funcional desses indivíduos. Objetivo: Correlacionar a capacidade funcional com a qualidade de vida de pacientes idosas antes e após a execução de programa de treinamento resistido (TR). Métodos: Foram avaliadas 15 idosas, com hipertensão controlada. O treinamento foi realizado durante um período de oito semanas. A qualidade de vida foi avaliada por meio do questionário SF-36. Resultados: Foram elegíveis 10 idosas hipertensas (70,7 $\pm 7,4$ anos). Observou-se correlação positiva moderada entre a distância percorrida e os domínios do questionário SF-36 antes e após treinamento, respectivamente: capacidade funcional $(\mathrm{r}=0,6)$, limitação por aspectos físicos $(r=0,5)$ e vitalidade, $(r=0,5)$. Conclusão: Houve associação entre a distância percorrida no TC6M e aqueles domínios do SF-36 que expressam a capacidade funcional.
\end{abstract}

Descritores: Envelhecimento; Exercício; Hipertensão; Qualidade de vida.

\begin{abstract}
Introduction: Hypertension is a chronic degenerative disease that influences the functional fitness and quality of life of elderly, the distance walked during the 6-minute walk advocated as an alternative for assessment of functional fitness of those patients. Methods: We evaluated 15 elderly women with controlled hypertension. The training was conducted over a period of eight weeks. Quality of life was assessed using the SF-36. Results: There were 10 eligible elderly hypertensive (70.7 \pm 7.4 years). There was a significant correlation between functional fitness and the domains of the SF-36 before and after training, respectively: physical functioning, role limitations due to physical and vitality. Conclusion: There was a relationship between the distance walked in $6 \mathrm{MWT}$ and those domains of the SF-36, which express functional capacity.
\end{abstract}

Key words: Aging; Exercise, Hypertension; Quality of life. 


\section{Introdução}

A hipertensão arterial sistêmica (HAS) configura-se como um dos agravos crônicos mais comuns e com repercussões clínicas graves. Estima-se que em todo o mundo 7,1 milhões de pessoas morram anualmente por pressão sanguínea elevada ${ }^{1,2}$. A HAS apresenta-se ainda como a principal doença crônica na população idosa, com uma prevalência de $65 \%$, sendo que entre as mulheres com mais de 65 anos, essa prevalência pode chegar a $80 \% \%^{2,3}$.

Alguns estudos ${ }^{3,4,5}$ revelam associação entre o declínio na habilidade funcional na vigência do aumento na pressão arterial sistólica, dessa forma, pacientes hipertensos têm risco aumentado de desenvolver incapacidade funcional, quando comparados a indivíduos normotensos.

O comprometimento da capacidade funcional do idoso tem implicações importantes para a família, a comunidade, para o sistema de saúde e para a vida do próprio idoso, uma vez que a incapacidade ocasiona maior vulnerabilidade e dependência, contribuindo para a diminuição do bem-estar e da qualidade de vida dos idosos $^{3,6}$.

Nos últimos anos, o treinamento resistido (TR) vem sendo citado em diversos estudos como uma alternativa segura e eficaz de prover uma melhoria na força e resistência musculares, capacidade funcional, e qualidade de vida, reduzindo a incapacidade de pessoas com e sem doença cardiovascular ${ }^{7,8}$.

Por ser ainda uma atividade de fácil controle de intensidade, e quando realizado de forma correta, o TR apresenta boa margem de segurança cardiológica, permitindo assim que idosos hipertensos se beneficiem dessa prática, uma vez que a literatura atual fornece dados que indicam a redução dos níveis de pressão arterial em indivíduos hipertensos ${ }^{9,10,11}$.

No entanto, apesar dos inúmeros benefícios que o TR proporciona, a literatura carece de trabalhos que avaliem os efeitos do TR sobre a capacidade funcional, bem como sua relação com a qualidade de vida em pacientes idosas e hipertensas. Dessa forma, neste estudo, objetivou-se verificar se há correlação entre o nível de capacidade funcional e a qualidade de vida em idosas hipertensas submetidas a programa de treinamento resistido.

\section{Materiais e métodos}

\section{Seleção da amostra}

A amostra foi composta por idosas com diagnóstico de HAS controlada recrutadas a partir do Programa de Apoio e Cuidado a Hipertensão Arterial (PACHA), o qual é vinculado a um hospital de alta complexidade em cardiologia.

Foram elegíveis os casos de idosas com idade igual ou superior a 60 anos, estabilidade clínica, autorização médica para participar do estudo com base na avaliação clínica e teste ergométrico (TE) prévios. Foram excluídas do estudo as pacientes que apresentaram resposta inapropriada da pressão arterial ou da frequência cardíaca durante o (TE), arritmias ventriculares induzidas pelo TE, hipertensão pulmonar grave ou outra doença pulmonar grave, consumo de bebida alcoólica e/ou tabaco, utilização de calmantes ou sedativos, confusão ou demência, limitação ortopédica e/ou déficit cognitivo que pudessem dificultar a execução dos testes, dor ou incapacidade para realizar o protocolo estabelecido, alteração na medicação durante o período da pesquisa, número de faltas maior que $15 \%$ do intervalo de tempo proposto para treinamento ou três faltas consecutivas, para que dessa forma não haja viés na avaliação ao final do treinamento, sendo mantido de todas as participantes o condicionamento obtido com o programa de TR.

Previamente, todas as participantes foram informadas quanto à finalidade do estudo, sendo solicitada a aquiescência com a assinatura do termo de consentimento aprovado pelo Comitê de Ética da instituição sob o número 223/08. Os procedimentos realizados atenderam às Diretrizes e Normas Regulamentadoras de Pesquisas 
Envolvendo Seres Humanos (Resolução 196/96 do Conselho Nacional de Saúde).

\section{Procedimentos de coleta de dados}

Antes da inclusão no programa de treinamento resistido, as participantes foram submetidas a uma avaliação inicial que se constituiu de anamnese médica, seguida de exame físico na qual foi realizada mensuração do peso corporal, estatura, cálculo do índice de massa corpórea (IMC), aferição da pressão arterial e frequência cardíaca. Uma vez cumprida essa etapa, as idosas foram encaminhadas ao serviço de ergometria da instituição para a realização do TE. Uma semana após a realização do TE as participantes foram submetidas ao teste de caminhada de seis minutos (TC6M) e responderam ao questionário Medical Outcomes Study 36-Item Short-Form Health Survey: SF-36 ${ }^{12}$.

Após o término do programa de TR, os procedimentos de avaliação inicial foram refeitos pelo mesmo avaliador.

\section{Teste de caminhada de seis minutos}

O TC6M foi realizado no período matutino, em um percurso de 30 metros, por um único examinador, seguindo o protocolo proposto pela American Thoracic Society (ATS) ${ }^{13}$. As pacientes foram orientadas a caminhar o mais rápido possível, sem correr, de acordo com sua tolerância ao exercício no período de 6 minutos. O examinador não impôs seu ritmo a paciente. Frases de incentivo foram pronunciadas durante a caminhada. Antes do início de cada teste, foram obtidas a frequência respiratória, a frequência cardíaca medida por oxímetro de pulso da marca Nonin ${ }^{\circledR}$ - modelo Onyx-9500 e a pressão arterial medida por esfigmomanômetro da marca $\mathrm{BD} \AA$ e estetoscópio da marca Littman ${ }^{\circledR}$, bem como a percepção de esforço por meio da Escala de Borg ${ }^{14}$. Ao término de cada teste, registraramse novamente os mesmos parâmetros. O resulta- do final do TC6M foi a medida da distância total percorrida em metros durante 6 minutos.

\section{Avaliação da qualidade de vida}

$\mathrm{Na}$ avaliação da qualidade de vida (QV) foi utilizado o questionário Medical Outcomes Study 36-Item Short-Form Health Survey (SF-36), validado no Brasil em 199912. Trata-se de formulário genérico que quantifica objetivamente a QV medindo aspectos de saúde e atividades geralmente afetadas pelas condições de saúde. É dividido em oito domínios em dois grandes componentes: o componente físico que envolve a capacidade funcional (CF), a dor, o estado geral de saúde (EGS) e o aspecto físico (AF) e o mental que contempla a saúde mental (SM), o aspecto emocional (AE), o aspecto social (AS) e a vitalidade (V), como descrito na Tabela1. O escore de cada domínio varia entre 0 a 100, no qual zero corresponde ao pior estado geral de saúde, e 100, ao melhor estado de saúde.

Apesar de esse instrumento ser autoadministrável, optou-se por aplicá-lo sob a forma de entrevista individualizada, devido à variabilidade escolar das voluntárias.

Tabela 1: Domínios do SF36 e suas respectivas abrangências

\begin{tabular}{|c|c|c|}
\hline & Domínios & Abrangência \\
\hline $\begin{array}{l}\text { Componente } \\
\text { físico }\end{array}$ & $\begin{array}{c}\text { Capacidade } \\
\text { funcional } \\
\text { Aspecto físico } \\
\text { Dor } \\
\text { Estado geral de } \\
\text { saúde }\end{array}$ & $\begin{array}{c}\text { Presença de limitação } \\
\text { física } \\
\text { Limitação de } \\
\text { atividades diárias } \\
\text { Intensidades e } \\
\text { limitações } \\
\text { Autopercepção de } \\
\text { saúde }\end{array}$ \\
\hline $\begin{array}{c}\text { Componente } \\
\text { mental }\end{array}$ & $\begin{array}{c}\text { Vitalidade } \\
\text { Aspecto social } \\
\text { Aspecto } \\
\text { emocional } \\
\text { Saúde mental }\end{array}$ & $\begin{array}{c}\text { Fraqueza e cansaço } \\
\text { Relacionamentos } \\
\text { Interferência } \\
\text { emocional } \\
\text { Depressão e ansie- } \\
\text { dade }\end{array}$ \\
\hline
\end{tabular}

\section{Programa de treinamento resistido}

Previamente ao TR, as voluntárias foram submetidas a um período de adaptação aos 
exercícios com duração de duas semanas, para aprendizado da técnica correta de execução dos movimentos. Durante esse tempo, os exercícios foram realizados sem carga.

Após o processo de adaptação, realizaramse testes de oito repetições máximas (8-RM) para os grupos musculares a serem treinados. O teste de 8-RM correspondeu à carga máxima que pode ser levantada pela participante em toda a amplitude normal do movimento com manutenção da técnica adequada, em oito repetições sucessivas.

O método de treinamento adotado foi o alternado por segmento com os exercícios feitos de forma sequencial na seguinte ordem: leg press, supino reto, cadeira extensora, puxada frontal, mesa flexora de joelho, abdução de ombro com halteres, abdução de quadril e rosca direta. A velocidade de execução utilizada foi 2:2 e o intervalo de recuperação de dois minutos entre cada série ${ }^{15}$.

Durante a execução dos movimentos, as pacientes foram orientadas a respirarem de forma adequada e continuamente no decorrer de cada repetição do exercício, expirando no momento da contração concêntrica e inspirando durante a contração excêntrica, evitando assim a chance de desenvolverem a manobra de Valsalva.

O TR consistiu no período de oito semanas, com uma frequência semanal de três vezes em dias alternados, realizado em duas séries de oito repetições, sempre à tarde. A intensidade do treinamento foi aumentada progressivamente ao longo do programa de treino, ou seja, ao final de cada semana a paciente era orientada a fazer mais duas repetições de cada exercício, e caso fosse possível, a carga atual elevava-se em $5 \%{ }^{16}$.

Antes do TR as pacientes realizavam cinco minutos de aquecimento, por meio de caminhada leve, seguida de autoalongamento dos principais músculos solicitados, o qual foi previamente orientado às pacientes. Após cada sessão de treinamento, foram repetidos os exercícios de autoalongamento.

\section{Anólise estatística}

Os dados foram analisados pelo software estatístico Statistical Package for Social Science versão 17.0 (SPSS Inc., Chicago, IL, USA). A análise descritiva foi apresentada em média e desvio-padrão (DP).

Inicialmente, foi verificada a normalidade das variáveis estudadas por meio do teste Kolmogorov-Smirnov (K-S), o qual indicou distribuição normal dos dados e assim permitiu a utilização de teste paramétrico. Dessa forma, utilizou-se o teste " $\mathrm{t}$ " de Student pareado para amostras dependentes na análise das variáveis presentes no SF-36 e na distância percorrida no TC6M, pré e pós o TR, bem como se utilizou o teste de correlação de Pearson na avaliação da associação entre a distância percorrida no TC6M e as demais variáveis presentes no SF-36, pré e pós-programa de TR. O nível de significância para o teste foi $5 \%$, com intervalo de confiança (IC) de $95 \%$ para todas as análises.

\section{Resultodos}

Entre novembro de 2009 a março de 2010, foram avaliadas 15 pacientes com diagnóstico de HAS, as quais apresentavam critérios de elegibilidade para este estudo. No entanto, obtevese uma perda de cinco participantes, pelos seguintes motivos: duas pacientes foram excluídas após avaliação ergométrica, e três desistiram de participar por motivos pessoais, dentre eles cirurgia de catarata, inviabilidade de condução até o local de treinamento e compromissos familiares. Dessa forma, a amostra foi composta de dez pacientes.

A média de idade para as pacientes submetidas ao TR foi 70,7 \pm 7,4 anos. As características gerais da população estudada encontram-se na Tabela 2. As medicações utilizadas pelas participantes também estão descritas nessa tabela. Durante este estudo não houve alteração dos medicamentos utilizados. 
O teste de caminhada de 6 minutos foi realizado sem intercorrências pelas idosas hipertensas. A média da distância percorrida em metros antes do treinamento foi de 418,5 $( \pm 58,50)$, pós-treinamento a média obtida foi de 436,1 ( $\pm 71,96)$, houve aumento na distância percorrida, porém esse aumento não foi significativo $(p=0,081)$.

Tabela 2: Características gerais da população estudada

\begin{tabular}{c|c}
\hline Idade (anos) & $70,7 \pm 7,4$ \\
\hline Peso (kg) & $61,6 \pm 9,04$ \\
\hline Altura (m) & $1,56 \pm 0,06$ \\
\hline IMC (kg/m $)^{2}$ & $25,14 \pm 3,34$ \\
\hline Variáveis hemodinâmicas (repouso) \\
\hline PAS (mmHg) & $150 \pm 15,63$ \\
\hline PAD (mmHg) & $94 \pm 8,43$ \\
\hline FC (bpm) & $78 \pm 18,67$ \\
\hline Comorbidades & $1(10 \%)$ \\
\hline Diabetes melito & $8(80 \%)$ \\
\hline Hipercolesterolemia & $1(25 \%)$ \\
\hline Osteoporose & $5(50 \%)$ \\
\hline Artrite & $5(50 \%)$ \\
\hline Obesidade & $4(40 \%)$ \\
\hline Medicações em uso & $6(60 \%)$ \\
\hline Betabloqueador & $1(10 \%)$ \\
\hline Bnibidor da enzima conversora de angiotensina & $4(40 \%)$ \\
\hline Diurético &
\end{tabular}

IMC - índice de massa corpórea; PAS - pressão arterial sistólica; PAD - pressão arterial diastólica; FC - frequência cardíaca.

A Tabela 3 exibe os escores dos domínios do SF-36 pré e pós-treinamento resistido. Após o programa de treinamento houve aumento na média dos escores em seis domínios desse instrumento, entretanto, não houve significância estatística.

Os domínios capacidade funcional, limitação por aspectos físicos, vitalidade e limitação por aspectos emocionais apresentaram correlação positiva moderada com a distância percorrida no TC6M antes e após programa de treinamento. No entanto, só houve correlação
Tabela 3: Valores de médias, desviospadrão e p valor das pontuações obtidas para cada domínio do questionório SF-36, em dez pacientes com diagnóstico de hipertensão arterial sistêmica

\begin{tabular}{c|c|c|c}
\hline Domínios & Pré TR & Pós TR & p valor \\
\hline \multicolumn{3}{c}{ Componente físico } \\
\hline $\begin{array}{c}\text { Capacidade } \\
\text { funcional }\end{array}$ & $80,5 \pm 19,64$ & $83 \pm 20,7$ & 0,72 \\
\hline Aspectos físicos & $75 \pm 33,33$ & $80 \pm 28,38$ & 0,55 \\
\hline Dor & $67,6 \pm 22,78$ & $68,5 \pm 34,68$ & 0,91 \\
\hline $\begin{array}{c}\text { Estado geral de } \\
\text { saúde }\end{array}$ & $75,65 \pm 21,58$ & $84,30 \pm 11,76$ & 0,15 \\
\hline \multicolumn{4}{c}{ Componente mental } \\
\hline Vitalidade & $80,50 \pm 14,61$ & $75,5 \pm 34,19$ & 0,61 \\
\hline Aspectos sociais & $83,75 \pm 23,60$ & $87,5 \pm 16,66$ & 0,61 \\
\hline $\begin{array}{c}\text { Aspectos } \\
\text { emocionais }\end{array}$ & $79,98 \pm 28,12$ & $89,90 \pm 16,13$ & 0,08 \\
\hline Saúde mental & $73,6 \pm 29,40$ & $72,40 \pm 24,76$ & 0,84 \\
\hline
\end{tabular}

TR: treinamento resistido; $p<0,05$.

significativa entre a distância percorrida no TC6M e os domínios vitalidade e limitação por aspectos emocionais pré-treinamento resistido, e pós-treinamento entre a distância percorrida e o domínio limitação por aspectos emocionais, como exposto na Tabela 4.

Tabela 4: Coeficiente de correlação entre os domínios do questionório SF-36 e a distôncia percorrida no TC6M antes e após TR

\section{Domínios do SF-36}

\begin{tabular}{c|c|c|c|c|c|c|c|c}
\hline $\mathbf{r d}$ & $\mathrm{CF}$ & $\mathrm{AF}$ & $\mathrm{DOR}$ & $\mathrm{EGS}$ & $\mathrm{V}$ & $\mathrm{AS}$ & $\mathrm{AE}$ & $\mathrm{SM}$ \\
\hline Pré-treino & 0,5 & 0,5 & 0,02 & 0,2 & $0,7^{*}$ & 0,4 & $0,6^{*}$ & 0,4 \\
\hline Pós-treino & 0,6 & 0,5 & 0,2 & 0,4 & 0,5 & $-0,2$ & $0,7^{*}$ & 0,2 \\
\hline
\end{tabular}

* $p<0,05$; CF - capacidade funcional; AF - aspectos físicos; EGS - estado geral de saúde; $V$ - vitalidade; AS - aspectos sociais; AE - aspectos emocionais; $S M$ - saúde mental; $r$ - coeficiente de correlação; d - distância percorrida no teste da caminhada de seis minutos.

\section{Discussão}

A literatura aponta a HAS como forte fator de risco para dependência funcional do idoso ${ }^{3,}$ 4,5 , neste estudo os valores obtidos no TC6M foram inferiores as médias obtidas em outros es- 
tudos que aplicaram o teste de caminhada em idosos saudáveis ${ }^{17,18}$.

A análise dos dados sugere que há uma tendência em incrementar a capacidade funcional após o TR pelo aumento na média da distância percorrida no TC6M após programa de TR, entretanto, esse acréscimo não obteve significância estatística provavelmente pelo reduzido número da amostra.

Apesar de não se ter encontrado um resultado expressivo neste trabalho, há várias pesquisas que confirmam os benefícios do TR na aptidão física em idosos devido principalmente, a aumentos na força e na potência muscular, as quais são importantes para a manutenção da independência funcional ${ }^{7,19,20}$.

Com relação à qualidade de vida mensurada por meio do questionário SF-36, observou-se pouco comprometimento nos domínios presentes nesse instrumento, contrariando alguns estudos que afirmam que indivíduos hipertensos tendem a ter menor $\mathrm{QV}^{21,22}$.

Uma possível explicação para esses resultados pode estar no fato da amostra possuir poucas comorbidades associadas, fato esse que encontra suporte em estudos que afirmam que pacientes com comorbidades associadas apresentam menor $\mathrm{QV}$, quando comparados com aqueles que não sofrem dessas complicações ${ }^{23,24}$. É possível também que a terapia medicamentosa anti-hipertensiva atual tenha promovido poucos efeitos colaterais, acarretando menor interferência na $\mathrm{QV}$ das idosas ${ }^{25,26}$.

Houve aumento na média dos valores de quase todos os domínios SF-36 após o programa de treinamento resistido, sugerindo melhoria na qualidade de vida propiciada pelo exercício físi$\mathrm{CO}$, fato esse que possui suporte pela literatura ${ }^{27,28}$.

A correlação moderada positiva entre a distância percorrida e o SF-36 ocorreu principalmente nos componentes físicos desse questionário, o que demonstra a intrínseca relação entre a capacidade funcional e qualidade de vida, o que implica dizer que o aumento na capacidade funcional proporcionado pelo treinamento resistido, possibilita melhorias na qualidade de vida nas idosas hipertensas ${ }^{29}$.
Entretanto, a única correlação significativa ocorreu no domínio aspectos emocionais antes e após treinamento. Esse mesmo domínio foi o que obteve maiores escores na avaliação da QV, resultado esse que pode ser atribuído à participação das pacientes em um programa de apoio a portadoras de HAS, que por fornecer educação em saúde com participação de uma equipe multiprofissional, propicia maior segurança no enfrentamento da doença e contribuiu para um bom equilíbrio emocional ${ }^{30}$.

Apesar dos resultados serem positivos, não houve significância estatística entre as médias obtidas no TC6M e todos os domínios do questionário SF-36, revelando a necessidade da realização de estudos com amostragem maior. Outro aspecto relevante seria a utilização de um questionário específico de qualidade de vida para a população de hipertensos em detrimento a utilização do questionário genérico.

\section{Conclusão}

Neste estudo, observou-se que houve correlação positiva moderada entre a distância percorrida no TC6M e os domínios do SF-36 que expressam a capacidade funcional do indivíduo, sugerindo que a melhora na capacidade funcional pode vir acompanhada de melhorias na qualidade de vida.

\section{Referências}

1. Passos VMA, Assis TD, Barreto SM. Hipertensão arterial no Brasil: estimativa de prevalência a partir de estudos de base populacional. Epidemiol Serv Saúde. 2006;15(1):35-45.

2. Sociedade Brasileira de Cardiologia. V Diretrizes Brasileiras de Hipertensão arterial. Arq Bras Cardiol. 2007;89(3):e24-e79.

3. Alves LC, Leimann BCQ, Vasconcelos MEL, Carvalho MS, Vasconcelos AGG, Fonseca TC O da. et al. A influência das doenças crônicas na capacidade funcional dos idosos do Município de São Paulo, Brasil. Cad Saúde Pública. 2007;23(8):1924-30. 
4. Hajjar I, Lakland DT, Cupples A, Lipsitz LA. Association between concurrent and remote blood pressure and disability in older adults. Hypertension. 2007;50:1026-1032.

5. Pedrosa R, Holanda G. Correlação entre os testes da caminhada, marcha estacionária e TUG em hipertensas idosas. Rev Bras Fisioter. 2009;13(3):252-6.

6. Arbex FS, Almeida EA. Qualidade de vida e hipertensão arterial no envelhecimento. Rev Soc Bras Clín Méd. 2009;7(5):339-42.

7. Chodzko-Zajko WJ, Proctor DN, Fiatarone Singh MA, Minson CT, Nigg CR, Salem GJ, et al. American College of Sports Medicine position stand. Exercise and physical activity for older adults. Med Sci Sports Exerc. 2009;41(7):1510-30.

8. Williams MA, Haskell WL, Ades PA, Amsterdam EA, PA, Bittner V, Franklin BA et al. Resistance exercise in individual with and without cardiovascular disease: 2007 update. Circulation. 2007;116:572-84

9. Polito MD, Farinatti PTV. Respostas de freqüência cardíaca, pressão arterial e duplo-produto ao exercício contra-resistência: uma revisão da literatura. Rev Port Ciênc Desporto. 2003;3(1):79-91.

10. Cornelissen VA, Fagard RH. Effect of resistance training on resting blood pressure: a meta-analysis of randomized controlled trial. J Hypertens. 2005;23(2):251-9.

11. Scher LML, Nobre F, Lima NKC. O papel do exercício físico na pressão arterial de idosos. Rev Bras Hipertens. 2008;15(4):288-31.

12. Ciconelli RM. Tradução para língua portuguesa e validação do questionário genérico de avaliação de qualidade de vida SF-36. Rev Bras Reumatol. 1999;39(3):143-50.

13. American Thoracic Society. Guidelines for the six-minute walk test. Am J Respir Crit Care Med. 2002;166:111-7.

14. Borg G. Escalas de Borg para a dor e o esforço percebido. São Paulo: Manole; 2000.

15. Terra DF, Mota MR, Rabelo HT, Bezerra LMA, Lima RM, Ribeiro AG et al. Redução da pressão arterial e do duplo produto de repouso após treinamento resistido em idosas hipertensos. Arq Bras Cardiol. 2008;91(5):299-305

16. Bird SP, Tarpening KM, Marino FE. Designing resistance training programmes to enhance muscular fitness: a review of the acute programme variables. Sports Med. 2005;35(10):841-51.

17. Barata VF, Gastaldi AC, Mayer AF, Sologuren MJJ Avaliação das equações de referência para predição da distância percorrida no tese de caminhada de seis minutos em idosos saudáveis brasileiros. Rev Bras Fisioter. 2005;9(2):165-71.
18. Pires SR, Oliveira AC, Parreira VF, Brito RR. Teste de caminhada de seis minutos em diferentes faixas etárias e índices de massa corporal. Rev Bras Fisioter. 2007;11(2):147-51.

19. Hunter GR, McCarthy JP, Bamman MM. Effects of resistance training on older adults. Sports Med. 2004;34(5):329-48.

20. Geraldes AAR, Dias Júnior NM, Albuquerque RB, Carvalho J, Farinnatti PTV. Efeitos de um programa de treinamento resistido com volume e intensidade moderados e velocidade elevada sobre o desempenho funcional de mulheres idosas. Rev Bras Ciênc Mov. 2007;15(2):53-60.

21. Brito DMS. Qualidade de vida e percepção da doença entre portadores de hipertensão arterial. Cad Saúde Pública. 2008;24(4):933-40.

22. Soni RK, Porter AC, Lash JP, Unruh ML. Health related quality of life in hypertension, chronic kidney disease, and coexistent chronic health conditions. Adv Chronic Kidney Dis. 2010;17(4):17-26.

23. Gusmão JL, Mion Jr. D, Pierin AMG. Healthrelated quality of life and blood pressure control in hypertensive patients with and without complications. Clinics. 2009;64(7):619-28.

24. Alonso J. Ferrer M, Gandek B, Ware JE, Aaronson NK, Mosconi P, et al. Health-related quality of life associated with chronic conditions in eight countries: results from the International Quality of Life Assessment (IQOLA) Project. Qual Life Res. 2004;13(2):283-98.

25. Ogihara T, Kuramoto K. Effect of long-term treatment with antihypertensive drugs on quality of life of elderly patients with hypertension: a double-blind comparative study between a calcium antagonist and a diuretic. Hypertens Res. 2000 Jan;23(1):33-7.

26. Handler J. Quality of life and antihypertensive drug therapy. J Clin Hypertens. 2005; 7(5):274-85.

27. Rocha CAQ, Paixão JA, Jucher G, Botaro CA, Bruno RX. Efeitos de um programa de força e resistência muscular na qualidade de vida de idosos. Brazilian Journal of Biomotricity. 2009;3(3):271-80.

28. Katula JA; Rejeski WJ; Marsh AP. Enhancing quality of life in older adults: a comparison of muscular strength and power training. Health and Quality of Life Outcomes. 2008;45(6):1-8.

29. Aragão JCB; Dantas EHM; Dantas, BHA. Efeitos da resistência muscular localizada visando a autonomia funcional e a qualidade de vida do idoso. Fitness \& Performance Journal. 2002;1(3):29-37.

30. Silqueira SMF. O questionário genérico SF-36 como instrumento de mensuração da qualidade de vida relacionada a saúde de pacientes hipertensos [tese]. Ribeirão Preto: Universidade de São Paulo; 2005. 